\title{
Occurrence of Hippocampal Ripples is Associated with Activity Suppression in the Mediodorsal Thalamic Nucleus
}

\author{
Mingyu Yang, ${ }^{1}$ Nikos K. Logothetis, ${ }^{1,2}$ and Oxana Eschenko ${ }^{1}$ \\ ${ }^{1}$ Max Planck Institute for Biological Cybernetics, Tübingen D-72076, Germany, and ${ }^{2}$ Centre for Imaging Sciences, Biomedical Imaging Institute, \\ The University of Manchester, Manchester M13 9PT, United Kingdom
}

Forming reliable memories requires coordinated activity within distributed brain networks. At present, neural mechanisms underlying systems-level consolidation of declarative memory beyond the hippocampal-prefrontal interactions remain largely unexplored. The mediodorsal thalamic nucleus (MD) is reciprocally connected with the medial prefrontal cortex (mPFC) and also receives inputs from parahippocampal regions. The MD may thus modulate functional connectivity between the hippocampus and the mPFC at different stages of information processing. Here, we characterized, in freely behaving Sprague Dawley male rats, the MD neural activity around hippocampal ripples, indicators of memory replay and hippocampal-cortical information transfer. Overall, the MD firing rate was transiently $(0.76 \pm 0.06 \mathrm{~s})$ decreased around ripples, with the MD activity suppression preceding the ripple onset for $0.41 \pm 0.04 \mathrm{~s}$ (range, 0.01- $0.95 \mathrm{~s}$ ). The degree of MD modulation correlated with ripple amplitude, differed across behavioral states, and also depended on the dynamics of hippocampal-cortical population activity. The MD suppression was the strongest and the most consistent during awake ripples. During non-rapid eye movement sleep, MD firing rate decreased around spindle-uncoupled ripples, but increased around spindle-coupled ripples. Our results suggest a competitive interaction between the thalamocortical and hippocampal-cortical networks supporting "on-line" and "off-line" information processing, respectively. We hypothesize that thalamic activity suppression during spindle-uncoupled ripples is favorable for memory replay, as it reduces interference from sensory relay. In turn, the thalamic input during hippocampal-cortical communication, as indicated by spindle/ripple coupling, may contribute to selectivity and reliability of information transfer. Both predictions need to be tested in future experiments.

Key words: cortical state; hippocampus; mediodorsal thalamus; memory consolidation; sharp-wave ripples; sleep

Significance Statement

Systems mechanisms of declarative memory consolidation beyond the hippocampal-prefrontal interactions remain largely unexplored. The connectivity of the mediodorsal thalamic nucleus (MD) with extrahippocampal regions and with medial prefrontal cortex underlies its role in execution of diverse cognitive functions. However, little is known about the MD involvement in "off-line" consolidation. We found that MD neural activity was transiently suppressed around hippocampal ripples, except for ripples co-occurring with sleep spindles, when the MD activity was elevated. The thalamic activity suppression at times of spindle-uncoupled ripples may be favorable for memory replay, as it reduces interference with sensory relay. In turn, the thalamic input during hippocampal-cortical communication, as indicated by spindle/ripple coupling, may contribute to selectivity and reliability of information transfer.

\section{Introduction}

Higher-order brain functions rely on fine-tuned interactions within large-scale brain networks. The network-level information processing takes place during alert behaviors, but also during socalled "off-line" states when sensory input is absent. The hippocam-

Received July 25, 2018; revised Oct. 1, 2018; accepted 0ct. 20, 2018.

Author contributions: M.Y. wrote the first draft of the paper; N.K.L. and 0.E. edited the paper; 0.E. designed research; M.Y. performed research; N.K.L. contributed unpublished reagents/analytic tools; M.Y. analyzed data; 0 .E. wrote the paper.

We thank Michel Beserve and Masataka Watanabe for valuable comments on the manuscript; Katalin Kalya for help with histology; and Eduard Krampe, Axel Oeltermann, and Joachim Werner for technical support. This research was supported by the Max Planck Society.

The authors declare no competing financial interests. pal-cortical network is essential for the encoding, consolidation, and retrieval of declarative memory (Wang and Morris, 2010; Eichenbaum, 2017). The two-stage model of the hippocampaldependent memory consolidation (Buzsáki, 1989) postulates that experience-induced changes of neural activity in the hippocampus (HPC) are mediated "on-line" by theta rhythm and "off-line" by high-frequency ( $\sim 200 \mathrm{~Hz})$ oscillations, or ripples, produced by synchronized discharge of CA1 neurons (Ylinen et al., 1995; Chrobak and Buzsáki, 1996). The hippocampal ripples preferentially occur

Correspondence should be addressed to Dr. Oxana Eschenko, Max Planck Institute for Biological Cybernetics, Max-Planck-Ring 8, Tubingen D-72076, Germany. E-mail: oxana.eschenko@tuebingen.mpg.de. https://doi.org/10.1523/JNEUROSCI.2107-18.2018

Copyright $\odot 2019$ the authors $\quad 0270-6474 / 19 / 390434-11 \$ 15.00 / 0$ 
during periods of enhanced cortical excitability (Sirota et al., 2003; Battaglia et al., 2004; Mölle et al., 2006) and coincide with thalamocortical sleep spindles (Siapas and Wilson, 1998). The rippleassociated replay of experience-activated neurons is thought to reflect memory trace reactivation and promote selective synaptic plasticity (Skelin et al., 2018). The coordinated hippocampal and cortical population activity is thought to mediate information transfer from the HPC to cortex for long-term storage (Buzsáki, 1989; Wang and Morris, 2010; Skelin et al., 2018).

The hippocampal-prefrontal pathway is the most extensively studied circuit in the context of mechanisms of declarative memory. Clearly, memory supporting network is not limited to these two brain regions; yet, the neural interactions beyond the hippocampal-prefrontal pathway remain largely unexplored. Our fMRI-based mapping of the whole brain activity at times of ripples in macaques revealed a characteristic pattern of positive and negative BOLD responses (Logothetis et al., 2012). Specifically, many cortical and limbic regions were activated during ripples, while a subset of subcortical areas, including the thalamus, showed activity suppression (Logothetis et al., 2012). Subsequent electrophysiological examination confirmed the ripple-associated inhibition in the lateral geniculate nucleus (Logothetis, 2015). The results of our fMRI study are consistent with numerous reports about tight temporal coupling between the population bursts in the HPC and neuronal activity in associative (Peyrache et al., 2009; Wierzynski et al., 2009; Wang and Ikemoto, 2016; Wilber et al., 2017) and primary sensory (Sirota et al., 2003; Ji and Wilson, 2007; Rothschild et al., 2017) cortices. Enhanced activity around ripples has been also shown in the ventral striatum (Lansink et al., 2008). Furthermore, bidirectional modulation was reported for neurons in the ventral tegmental area (VTA; Gomperts et al., 2015) and basolateral amygdala (BLA; Girardeau et al., 2017), while activity suppression was found in the median raphe (Wang et al., 2015) and the midline thalamic nuclei (LaraVásquez et al., 2016).

In the present study, we sought to characterize the rippleassociated activity in the mediodorsal thalamic nucleus (MD). The MD integrates various types of information and mediates it to the medial prefrontal cortex (mPFC) for execution of diverse cognitive functions (Mitchell, 2015), including memory (Markowitsch, 1982; Van Der Werf et al., 2003). Although there are no known direct projections between the MD and HPC (Groenewegen, 1988; Varela et al., 2014), the MD contributes to declarative memory as a part of extrahippocampal circuit (Ketz et al., 2015). The MD receives afferents from the lateral entorhinal and perirhinal cortices, which provide parallel multisensory input to the MD and HPC (Groenewegen, 1988; Burwell, 2000). The MD is reciprocally connected with the $\mathrm{MPFC}$, which in turn receives direct input from the HPC (Jay et al., 1992). The MD is thought to gate the hippocampal-cortical and cortical-cortical interactions that are relevant for declarative memory (Floresco and Grace, 2003; Ketz et al., 2015). However, the role of MD in off-line processing has not been explored. Here, we report that the MD activity is systematically suppressed around ripples. We also found that the ripple-associated MD modulation varied across awake and sleep states. The MD suppression was more consistent and robust during awake state, while synergistic activation of the MD-HPC-mPFC network occurred during nonrapid eye movement (NREM) sleep at times of ripple/spindle coupling. Collectively, our results suggest competitive interactions between large-scale brain networks mediating different stages of information processing.

\section{Materials and Methods}

Animals. Eight adult male Sprague Dawley rats (Charles River Laboratories) weighting 300-450 g were used. After surgery rats were singlehoused and kept on a $12 \mathrm{~h}$ light/dark cycle (8:00 A.M. lights on). All the experiments were performed during dark cycle. The study was performed in accordance with the German Animal Welfare Act (TierSchG) and Animal Welfare Laboratory Animal Ordinance Tierschutz-Versuchstierverordnung (TierSchVersV), and was in full compliance with the guidelines of the EU Directive 2010/63/EU on the protection of animals used for scientific purposes. The study was reviewed by the ethics commission Tierschutzgesetz, (TierSchG, Section 15) and approved by the state authority (Regierungspräsidium, Tübingen, Baden-Württemberg, Germany).

Anesthesia. Implantation of electrodes was performed under isoflurane anesthesia (initiation 4\%, maintenance 1.5-2.0\%). The depth of anesthesia was controlled by ensuring a lack of responses to mildly noxious stimuli (pinch of hind paw). Heart rate and blood oxygenation were monitored using a pulse oximeter (Nonin $8600 \mathrm{~V}$, Nonin Medical); supplementary oxygen was provided to maintain the blood oxygenation level above $90 \%$. Body temperature was maintained at $37^{\circ} \mathrm{C}$ throughout the entire anesthesia period.

Surgery and electrode placement. A fully anesthetized rat was fixed in a stereotaxic frame with the head angle at $0^{\circ}$. The skull was exposed, and a local anesthetic (Lidocard 2\%, B. Braun) was applied on the skin edges to additionally numb the skin. Burr holes were made for electrodes and anchor screws. Dura mater was removed when necessary. For EEG recordings, a stainless-steel screw ( $0.86 \mathrm{~mm}$ diameter, Fine Science Tools) was placed above the frontal cortex, and the ground screw was placed above the cerebellum. Four anchor screws $(1.19 \mathrm{~mm}$ diameter, Fine Science Tools) were placed on the skull side edges. Screws were fixed in the skull and additionally secured with tissue adhesive. For extracellular recording in the MD and HPC, twisted wires or tube tetrodes (Kapoor et al., 2013) were used. The tetrodes were made from insulated nichrome wire (12 $\mu \mathrm{m}$ diameter, Sandvik Wire and Heating Technology) and assembled into a microdrive (Versadrive; Neuralynx). A custom design of the microdrive allowed targeting the MD with up to six tetrodes and dorsal HPC (dHPC) with up to two tetrodes. Before implantation, tetrode impedance was adjusted to $150-500 \mathrm{k} \Omega$ by gold plating. The drive with electrodes was fixed in a stereotaxic micromanipulator (David Kopf Instruments) and slowly lowered until the electrode tips targeting the MD reached $\sim 5.0 \mathrm{~mm}$ below the brain surface. The tips of dHPC electrodes were fixed $\sim 3.0 \mathrm{~mm}$ higher and expected to reach $\sim 2.0 \mathrm{~mm}$ depth. A single platinum-iridium electrode (FHC) was implanted in the mPFC. The final adjustment of the electrode position was guided by on-line monitoring of neural activity. The entire implant was secured on the skull with dental cement (Paladur, Heraeus Kulzer). A copper mesh was mounted around the implant to protect exposed wires and also to isolate recording electrodes from the electrical noise. The injection of analgesic $(2.5 \mathrm{mg} / \mathrm{kg}$, s.c.; Finadyne, Essex $)$ and antibiotic $(5.0 \mathrm{mg} / \mathrm{kg}$, s.c.; Baytril, Bayer) was given before rat awakening from anesthesia and repeated at $24 \mathrm{~h}$ intervals during next $4 \mathrm{~d}$. Animals were allowed 1 week of postsurgery recovery.

Electrophysiological recording. Rats were first habituated to the recording setup and cable plugging procedure. The head implant was connected to the Neuralynx Digital Lynx acquisition system via two 16-channel head stages with red/green tracking LEDs (Neuralynx). The electrode placement in the MD was optimized by lowering the electrodes in $0.05-$ $0.1 \mathrm{~mm}$ steps and monitoring spiking activity on the high-passed $(300 \mathrm{~Hz}$ to $8 \mathrm{kHz}$ ) extracellular signal. The depth of dHPC electrodes was adjusted by gradually lowering the tetrodes (maximum $0.05 \mathrm{~mm}$ per day) until reliable ripple activity was observed. Once the electrode position was optimized, the broad-band $(0.1 \mathrm{~Hz}$ to $8 \mathrm{kHz})$ extracellular signals were acquired and digitized at $32 \mathrm{kHz}$. The brain activity was referenced to the ground electrode. The animals' movement was monitored by video tracking (25 frames per second) with the aid of LEDs attached to the head stage. All recordings were performed between 10:00 A.M. and 7:00 P.M. for up to $2 \mathrm{~h}$.

Single-unit isolation. The MD single units were isolated using two or four tetrode channels. Extracellular signals were high-pass $(600 \mathrm{~Hz})$ fil- 
tered and the spike waveforms with negative peaks exceeding $-0.05 \mathrm{mV}$ were extracted. The template-matching algorithm based on the principal component analysis was used for clustering the spike waveforms of individual units (Spike2 software, Cambridge Electronic Design). Various specific measurements of the spike waveform were additionally used for cluster refinement. A unit cluster was classified as a single-unit activity (SUA) if the refractory period (time between two consecutive spikes) was at least $1 \mathrm{~ms}$. When the recording quality and the spike sorting did not allow unambiguous single-unit isolation, the spike cluster was conservatively classified as multiunit activity (MUA).

Classification of behavioral states. We classified the rat spontaneous behavior into awake and NREM sleep using frontal EEG or PFC local field potentials (LFPs) by applying a standard sleep-scoring algorithm described in detail previously (Novitskaya et al., 2016). Briefly, animal movement speed was extracted from the video recording synchronized with neural signal. The $\theta / \delta$ ratio was calculated from the artifact-free EEG in $2.5 \mathrm{~s}$ epochs. The epochs of awake state were identified by the presence of active locomotion and above threshold $\theta / \delta$ ratio; the epochs of NREM sleep were identified by the absence of motor activity and below threshold $\theta / \delta$ ratio. The minimal duration of the same behavioral state was set to $20 \mathrm{~s}$; data segments with less steady behavioral states were excluded from further analysis.

Detection of cortical spindles. Sleep spindles were detected from the artifact-free EEG channel by bandpass (12-16 Hz) filtering, downsampling to $200 \mathrm{~Hz}$, and thresholding of the spindle band power envelop (root mean square (RMS) with $0.2 \mathrm{~s}$ smoothing window; spindle RMS). The spindle detection threshold was calculated as 3 SDs of the spindle RMS during NREM sleep. The minimal length of sleep spindle was set to $0.5 \mathrm{~s}$ of continuous spindle RMS values above the threshold. The spindle onsets and offsets were defined at 1 SD threshold ascending and descending crossings, respectively.

Detection of hippocampal high-frequency oscillations. The transient high-frequency population bursts were detected from the LFPs recorded in the pyramidal layer of the dorsal CA1. The LFP signal was bandpass (gamma band, 70-120 Hz; ripple band, 120-250 Hz) filtered, rectified, and low-pass filtered at $25 \mathrm{~Hz}$. The resulting signal was $z$-score normalized. The high-frequency events were identified by signal amplitude exceeding a threshold of 5 SDs for ripples and 4 SDs for gamma events. The event onsets and offsets were defined at the 1 SD threshold ascending and descending crossings, respectively. We used a $70 \mathrm{~ms}$ refractory window to prevent double detection of the same event. The Morlet-wavelet timefrequency analysis was used to classify the events into gamma, gamma/ ripple, and ripple oscillations based on intraevent frequency. For exploring the temporal pattern of ripple occurrence, we used a method based on computing the logarithm of inter-ripple intervals (IRIs), described in detail previously (Selinger et al., 2007). Briefly, the bimodal $\log ($ IRI ) distribution indicated two temporal patterns of ripple occurrence with shorter and longer IRIs. We identified the crossing time of two distributions and used it for classifying "clustered" and "isolated" ripples. Ripples with short IRIs $(<0.33 \mathrm{~s})$ were considered as a ripple cluster and treated as a single ripple event. The time of the first ripple in the cluster was considered the ripple onset time. Spindle-coupled ripples were defined as ripples occurring between onsets and offsets of spindles.

Analysis of perievent neural activity. To examine neural activity around transient oscillatory events (ripples, spindles, gammas), perievent spike histograms (PETHs) were generated $\pm 2 \mathrm{~s}$ around the event onsets and smoothed with a Gaussian window (window size, $6 \mathrm{~ms}$; bin size, $2 \mathrm{~ms}$ ). To minimize the impact of slow fluctuations of the neuronal excitability that is synchronized throughout the entire forebrain, we applied an event permutation procedure. In specific, we created a "surrogate" event sequence by randomly distributing the same number of events detected every $4 \mathrm{~s}$ in a given session. The permutation procedure was repeated 100 times and the PETHs were generated $\pm 2 \mathrm{~s}$ around the surrogate events. The time series ( $2 \mathrm{~ms}$ bins) of MD MUA/SUA around the surrogate events were subtracted from the corresponding values around "true" events, and the resulting ("corrected") PETHs were $z$-score normalized to the MD MUA/SUA firing rate during the entire $4 \mathrm{~s}$ time window of the surrogate PETHs.
To quantify perievent changes of neural activity, a modulation index (MI) was calculated by extracting the area above/below the curve of each PETH $\pm 0.5 \mathrm{~s}$ around time 0 . To determine the significance of modulation, we built PETHs around surrogate events generated by permutation of the interevent intervals and calculated the MIs for each of 5000 shuffled PETHs. The 95\% confidence interval (CI) served as the significance threshold. The onset/offset and the duration of the firing rate change were defined by the $\pm 1 \mathrm{SD}$ threshold crossing of normalized perievent MD MUA. For examining MD modulation around subsets of ripples, for each data set, the ripple times were bootstrapped, and the bootstrapped MI (b-MI) was computed for 100 randomly selected ripples. Then the same procedure was repeated 5000 times, and the distribution of b-MIs was plotted, also for awake and NREM sleep ripples.

Experimental design and statistical analysis. The LFPs and MUA recordings from different placements in the MD were considered independent if a linear distance between the sites exceeded $140 \mu \mathrm{m}$ (Buzsáki, 2004). A one-way ANOVA was used for independent measures. In the case of repeated observations, a repeated-measures ANOVA or paired $t$ test was used when data met the criteria for normality; otherwise, a Wilcoxon signed-rank test was used. For ANOVAs, when the sphericity assumption was violated, the Greenhouse-Geisser correction method was applied. Bonferroni's test was used for post hoc comparisons. Pearson's linear correlations were used when appropriate. The statistical significance $\alpha$ value was set at $p=0.05$. The IBM SPSS Statistics (version 22) and MATLAB (MathWorks) software packages were used for statistical analysis.

Perfusion and histology. After the last recording session, rats were killed with a lethal dose of pentobarbital sodium (100 mg/kg i.p.; Narcoren, Merial) and perfused transcardially with $0.9 \%$ saline followed by $4 \%$ paraformaldehyde in $0.1 \mathrm{M}$ phosphate buffer (PB), pH 7.4. The brains were removed and stored in the same fixative. Before sectioning, whole brains were placed in $0.1 \mathrm{M} \mathrm{PB}$ buffer containing 30\% sucrose until they sank. Serial $60-\mu \mathrm{m}$-thick coronal sections were then cut on a horizontal freezing microtome (Microm HM 440E; Thermo Fisher Scientific, Waltham, MA), collected in $0.1 \mathrm{M} \mathrm{PB}$, and then directly stained or stored at $-20^{\circ} \mathrm{C}$ in a cryoprotectant solution (30\% ethylene glycol and $10 \%$ sucrose in $0.05 \mathrm{M} \mathrm{PB}$ ) until further processing. Nissl staining was performed according to a standard procedure. Briefly, sections were mounted on gelatin-coated glass slides, defatted, stained with cresyl violet, rinsed with acetic acid, dehydrated, and coverslipped. All sections were examined using an AxioPhot or AxioImager microscope (Carl Zeiss). Positions of the electrode tips were assessed visually and digitized.

\section{Results}

We obtained 42 simultaneous recordings in the CA1 area of the dHPC, prelimbic area of the PFC, and MD in eight rats. Figure $1 A$ illustrates the electrodes targeting the $\mathrm{HHPC}$ and MD. We sampled MD activity from different subregions of the MD using multiple tetrodes and by lowering tetrodes after each recording session (see Materials and Methods). Two to 17 different electrode placements per rat resulted in total of $49 \mathrm{MD}$ recordings from the medial $(n=19)$, central $(n=10)$, and lateral $(n=20)$ sections of the MD. Figure $1 B$ shows the reconstruction of the deepest recording sites in the MD. Preliminary analyses did not reveal any difference in neural activity across the MD sites; therefore, the results described below were obtained using combined data sets from different MD subregions, and, for simplicity, we will refer to all thalamic recordings as MD.

\section{Neural activity in the MD is suppressed around hippocampal ripples}

We first characterized the overall population activity in the MD around hippocampal ripples. Ripples were detected by thresholding at 5 SDs of the bandpass-filtered $(120-250 \mathrm{~Hz}$; smoothed at $25 \mathrm{~Hz}$ ), rectified, and $z$-score-normalized CA1 LFP signal (Fig. 2, 
A

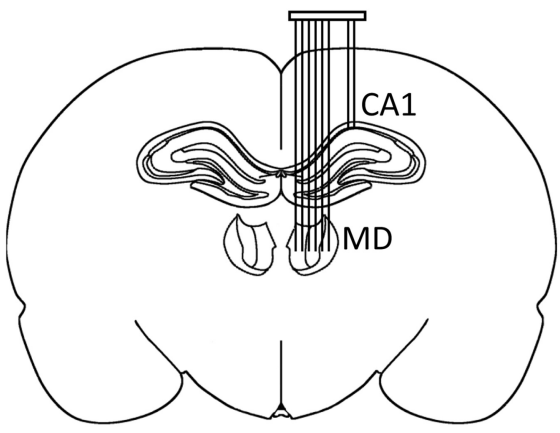

B
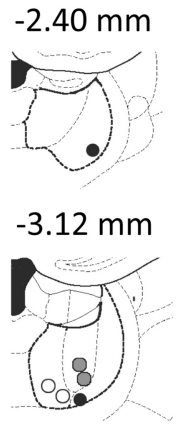

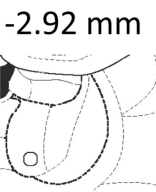

$-3.48 \mathrm{~mm}$

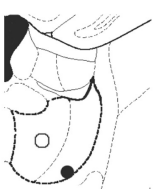

\section{$-3.00 \mathrm{~mm}$}

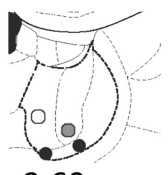

$-3.60 \mathrm{~mm}$

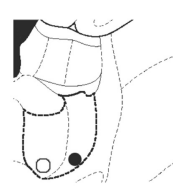

Figure 1. Simultaneous multisite electrophysiological recording in freely behaving rats. $A$, Schematic illustration of the electrode placements in the dHPC and MD. Tetrodes ( $2 \mathrm{for} \mathrm{dHPC}$ and up to 6 for MD) were mounted on a movable microdrive allowing depth adjustment of each tetrode individually. $\boldsymbol{B}$, Reconstruction of the deepest recording sites within the MD is shown on different anterior-posterior planes. White circles, Medial MD; gray circles, central MD; black circles, lateral MD.

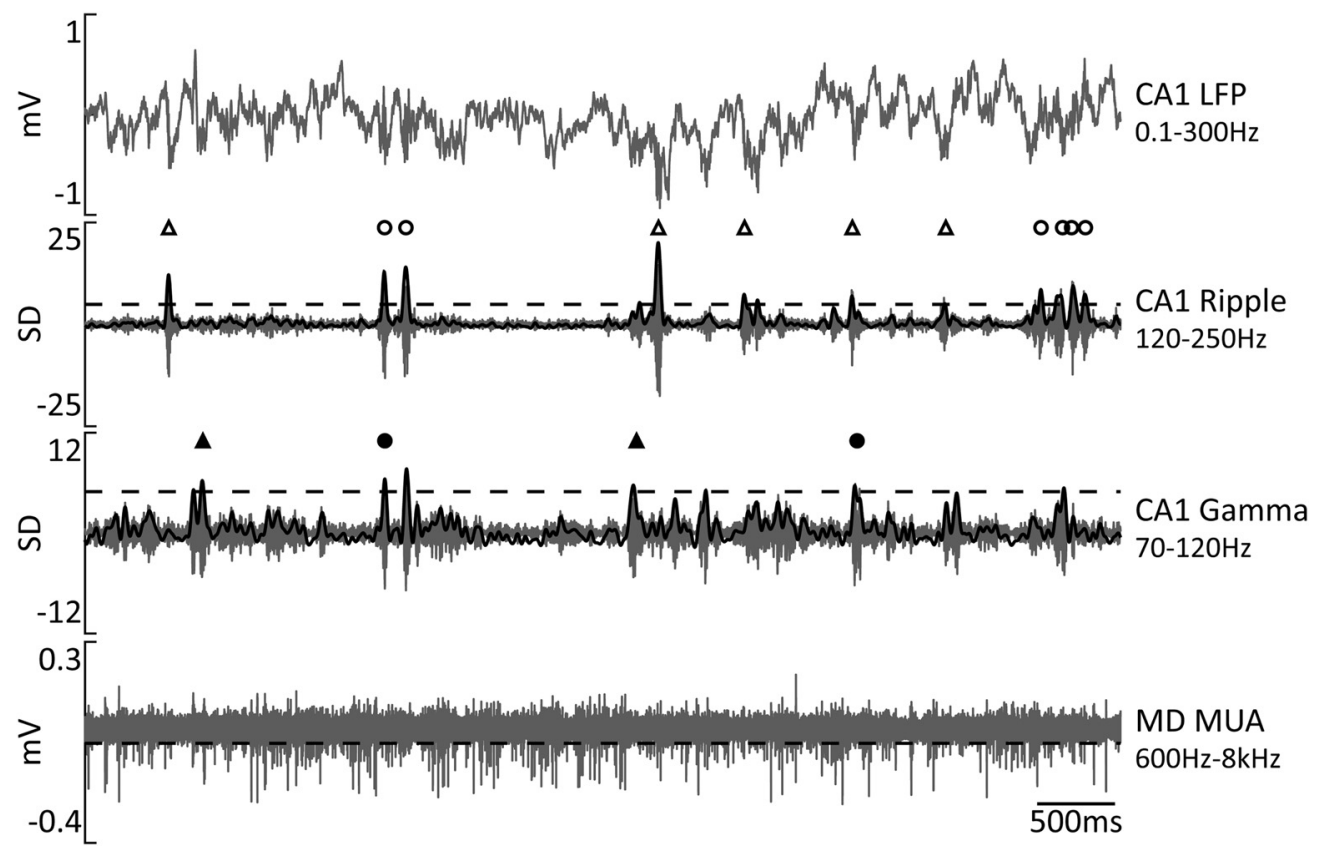

Figure 2. Detection of ripple and gamma oscillations. Representative traces of simultaneously recorded neural activity in the dHPC (top) and MD (bottom). The two middle traces show bandpass-filtered dHPC LFPs; black solid lines show rectified and smoothed signals. Horizontal dashed lines mark the detection threshold for ripples at 5 SDs, gamma events at 4 SDs, and spikes at $-0.05 \mathrm{mV}$. Symbols above two middle traces mark transient oscillatory events. Open triangles mark isolated ripples, open circles mark clustered ripples, black triangles mark gamma events, and black circles mark gamma/ripple events.

middle). On average, $1013.4 \pm 66.3$ ripples were detected per each recording session. The multiunit spike times (referred to here as multiunit activity) were extracted from the wide-band $(0.1 \mathrm{~Hz}-8 \mathrm{kHz})$ extracellular signal recorded in the MD by highpass $(600 \mathrm{~Hz})$ filtering and thresholding at $-0.05 \mathrm{mV}$ (Fig. 2, bottom). For each recording session and each MD site, a PETH was generated at $\pm 2 \mathrm{~s}$ around ripple onsets and subsequently corrected for spiking activity fluctuation around randomly distributed surrogate events (for details, see Materials and Methods). A significant transient suppression of the MD MUA around ripples was present in essentially all MD sites (Fig. $3 A$ ). We also calculated a modulation index (Fig. $3 C$ ) and tested each MD MUA case for significant modulation (see Materials and Methods). The periripple firing rate decrease was significant for all 49 MD MUA recordings (mean MI lower than the lower limit of $95 \%$ CIs). To characterize in more detail the dynamics of rippleassociated modulation of MD MUA, for each session-averaged PETH, we extracted the onset, peak time, and duration of the firing rate change, as illustrated in Figure 3C. Remarkably, in most cases, a decrease of the MD firing rate preceded the ripple onset, occurring, on average $0.41 \pm 0.04 \mathrm{~s}$ (range, 0.01-0.95 s) before the ripple. The MD suppression lasted, on average, for $0.76 \pm 0.06 \mathrm{~s}$ (range, $0.13-2.10 \mathrm{~s}$ ) and peaked at $0.11 \pm 0.01 \mathrm{~s}$ after ripple onset (range, $0.04-0.39 \mathrm{~s}$ ). Figure $3 D$ shows the distribution for the MD inhibition onset (red line), duration (blue), and times of the minimal firing rate (black). There was no difference in the onset, peak time, or duration of MD MUA suppression across different MD subregions (one-way ANOVA, onset, $F_{(2,37)}=0.72, p=0.50$; peak time, $F_{(2,37)}=0.14, p=0.87$; duration, $\left.F_{(2,37)}=0.36, p=0.70\right)$.

It is possible, however, that the multiunit population response does not capture the response profiles of the individual MD neurons. Therefore, we also characterized the ripple-associated firing rate modulation of the MD single units. The recording quality permitted reliable isolation of 40 single units ( $n=6$ rats). The ripple-associated suppression was present in all MD single units (Fig. 3B). We thus considered the MD neurons as responding homogeneously and used MD MUA for further analyses. 
A

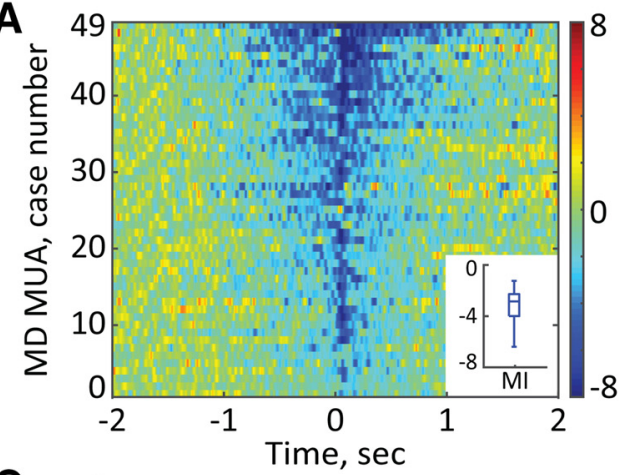

C

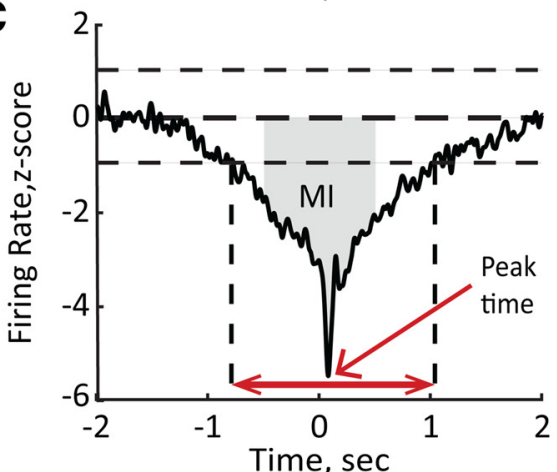

B

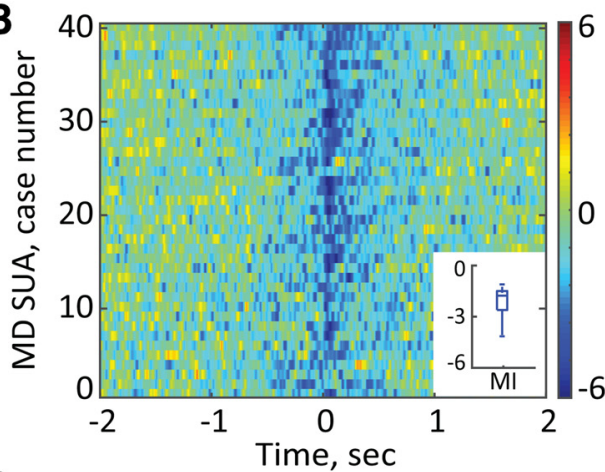

D

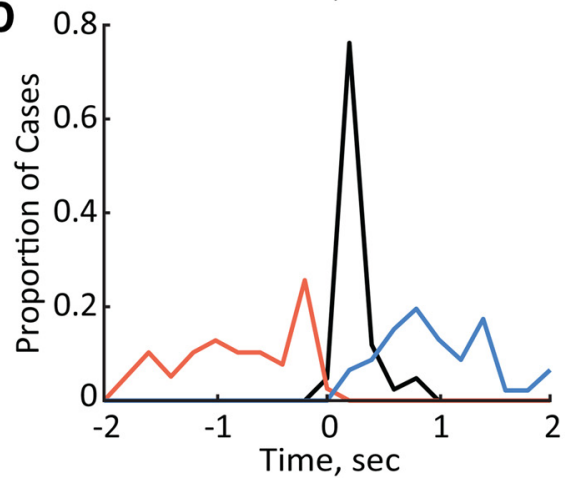

Figure 3. Ripple-associated suppression of the MD neural activity. $\boldsymbol{A}, \boldsymbol{B}$, Normalized firing rate is plotted for MD MUA ( $\boldsymbol{A}$ ) and MD single units ( $\boldsymbol{B}$ ). The averages around onsets of all detected ripples are shown for 49 MD MUA recordings obtained from different MD sites in eight rats and 40 single units ( $n=6$ rats). The cases are sorted according to the values of the MI. Color bars show z scores. Insets, Median and variability outside the upper and lower quartiles are indicated. $C$, Quantitative characterization of the ripple-associated firing rate change. The MI was calculated as the area (gray) between the curve and $y=0$ within $\pm 0.5 \mathrm{~s}$ around the ripple onset $(t=0)$. The onset and offset of modulation were detected by \pm 1 SD threshold crossings (vertical dashed lines). The time between onset and offset was considered the duration of modulation (red double-headed arrow). The peak time was determined as the time with the minimum firing rate (red arrow) $\boldsymbol{D}$, The distribution of onsets (red), peak times (black), and offsets (blue) of the MD firing rate change relative to ripple onset $(t=0)$ for all MD MUA cases $(n=49)$ shown in $\boldsymbol{A}$.

\section{The degree of MD suppression}

correlates with ripple features

We examined whether the degree of MD MUA modulation systematically varied with ripple intrinsic properties. To this end, we split all detected ripples according to their amplitude or intraripple frequency into quartiles and extracted for each ripple group the corresponding MI. A significant linear correlation was found between the MI and ripple amplitude $(r=-0.28, p<$ 0.0001); a stronger MD MUA suppression was associated with ripples of higher amplitude (Fig. 4A). No such relationships were revealed between $\mathrm{MI}$ and intraripple frequency $(r=0.096, p=0.18)$.

We also studied whether the MD modulation depended on the temporal pattern of ripple occurrence. The visual inspection of the bandpass-filtered $(120-250 \mathrm{~Hz})$ CA1 LFPs indicated that some ripples occur in close $(<0.33 \mathrm{~s})$ temporal proximity to each other, while other ripples are rather sparse (Figs. 2, middle, $4 B$ ). Thus, we classified the detected ripples as isolated or clustered (see Materials and Methods). Clusters typically consisted of two to seven ripples. We computed the PETHs of the MD MUA around isolated and clustered ripples (in the case of ripple clusters, the first ripple onset was used as $t=0$ ) and observed a much stronger MD suppression around isolated ripples $(-2.75 \pm$
A

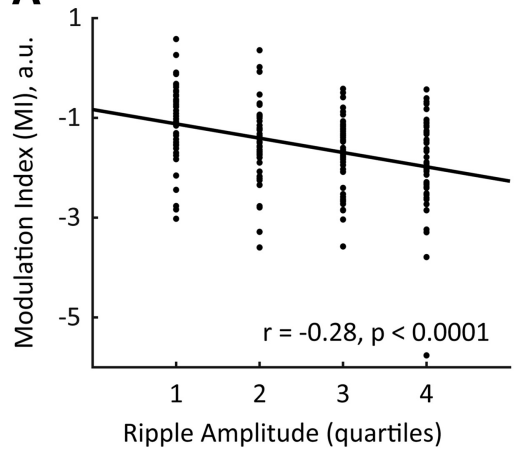

B

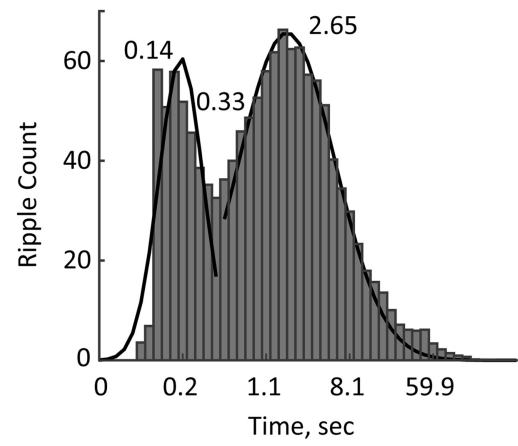

Figure 4. The degree of MD modulation correlated with ripple amplitude and the temporal pattern of ripple occurrence. $\boldsymbol{A}$, The Mls from all 49 MD MUA cases are plotted according to the quartiles of ripple amplitude. The black line shows the linear regression. Note that stronger MD suppression (lower Mls) corresponds to ripples of higher amplitude. $\boldsymbol{B}$. The log-scale distribution of interripple intervals with Gaussian fittings (black lines). Numbers indicate the peak times and the crossing time of two Gaussians. Note that there is a bimodal distribution of inter-ripple intervals.

0.19 vs $-1.94 \pm 0.11$ for MIs of isolated vs clustered ripples, respectively; paired $t$ test, $\left.t_{(40)}=4.12, p<0.001\right)$. Overall, the amplitude of isolated ripples was slightly lower than that of clustered ripples $(8.86 \pm 0.23$ vs $9.71 \pm 0.27 \mathrm{SDs}$, for isolated vs clustered ripples, respectively; paired $t$ test, $t_{(40)}=11.12, p<$ $0.0001)$. The difference in the temporal pattern of ripple occurrence did not affect the strong negative correlation between the $\mathrm{MI}$ and ripple amplitude, as such relationships were present for both types of ripples (isolated, $r=-0.24, p<0.001$; clustered, $r=-0.27, p<0.0001)$. 
A
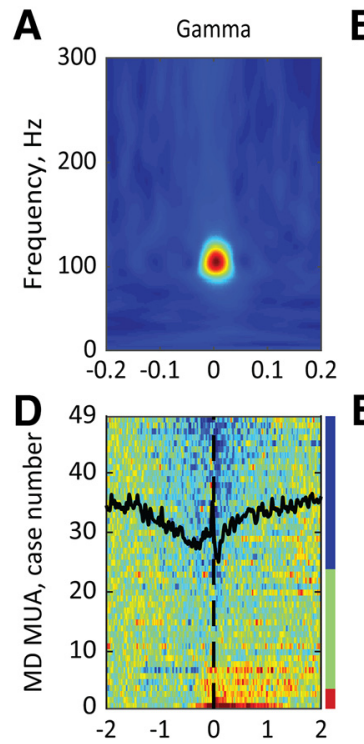

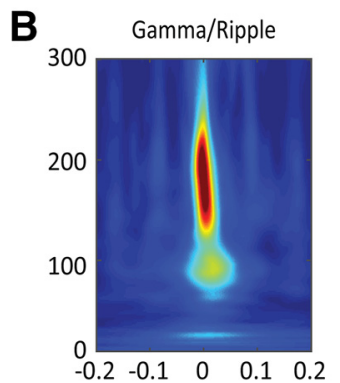

E

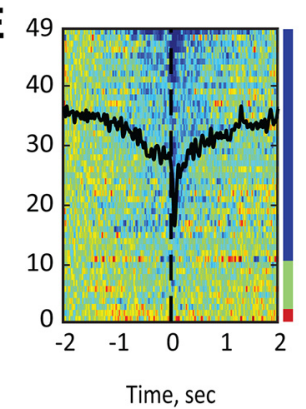

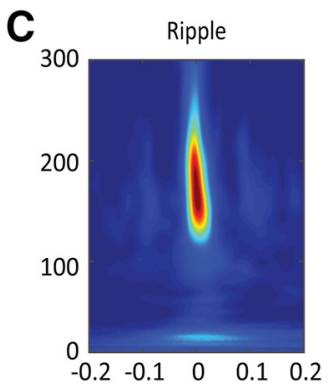

$\mathbf{F}$

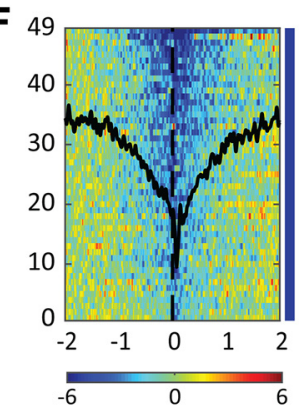

Figure 5. The MD activity modulation around different types of high-frequency population bursts in the $\mathrm{dHPC}$. $\boldsymbol{A}-\boldsymbol{C}$, Representative perievent spectrograms for gamma (left), gamma/ripple (middle), and ripple (right) events. $\boldsymbol{D}-\boldsymbol{F}$, Normalized and colorcoded MD firing rates are plotted for all MD MUA cases $(n=49)$ around the gamma $(\boldsymbol{D})$, gamma/ripple $(\boldsymbol{E})$, and ripple $(\boldsymbol{F})$ events. The horizontal color bar shows z scores. Overlaid black lines show the averaged MD population dynamics around corresponding event types. The color bar on the right of each plot shows the proportion of MD MUA cases with ripple-associated suppression (blue), ripple-associated activation (red), and no modulation (green). Note the strongest MD suppression around ripples and bidirectional changes of the MD firing rate around gamma and gamma/ripple events.

\section{Modulation of MD activity around gamma oscillations}

We also characterized the dynamics of MD MUA around transients of high-gamma $(70-120 \mathrm{~Hz})$ oscillations (or gamma events), which is another prominent type of population activity in the HPC (Sullivan et al., 2011). We detected the gamma events by thresholding at 4 SDs of the bandpass-filtered $(70-120 \mathrm{~Hz}$; smoothed at $25 \mathrm{~Hz}$ ), rectified, and $z$-score-normalized CA1 LFP signal (Fig. 2, middle). Consistent with the existing literature (Ramirez-Villegas et al., 2015), a substantial fraction of gamma events $(40.78 \pm 1.92 \%)$ co-occurred with ripples. Based on the spectral composition of the detected transient high-frequency oscillations, we split them into "pure" gamma, "mixed" gamma/ripple, and "pure" ripple events (Fig. 5A-C). The MD MUA was significantly suppressed around pure ripples in all cases $(n=49)$, while around gamma/ripple and pure gamma events, the MD MUA decrease was detected in $79.6 \%(n=39)$ and $53.1 \%(n=26)$ of cases, respectively. Consequently, the mean MI was the lowest for pure ripples $(-2.87 \pm 0.15)$, indicating a more consistent decrease of the MD firing rate, compared to other event types $(-1.81 \pm 0.18$ and $-1.42 \pm 0.13$ for gamma/ ripple and pure gamma events, respectively). Figure $5 D-F$ shows the profiles of the MD firing rate for each event type. Finally, we compared the magnitude of MD suppression across event types by submitting only significantly modulated cases to repeated-measures ANOVA. There was a significant main effect of the event type $\left(F_{(2,62)}=31.99, p<0.0001\right)$. Post hoc tests showed that the MD MUA suppression was the strongest around pure ripples and the weakest around pure gamma events. Based on this result, for further analysis we considered only ripple oscillations without accompanying gamma power increase.
Ripple-associated modulation of MD activity depends on behavioral state We further explored whether the rippleassociated modulation of MD MUA depended on the behavioral state. We classified the behavioral state as awake or NREM sleep using frontal EEG or PFC LFPs (see Materials and Methods), as also described in detail previously (Novitskaya et al., 2016). Briefly, animal movement speed was extracted from the video recording synchronized with the neural signal. The $\theta / \delta$ ratio was calculated from the artifact-free EEG in 2.5 s epochs. The epochs of awake state were identified by the presence of active locomotion and an above-threshold $\theta / \delta$ ratio; the epochs of NREM sleep were identified by the absence of motor activity and a belowthreshold $\theta / \delta$ ratio. Consistent with the existing literature (Llinás and Steriade, 2006), MD activity was higher during awake state than during NREM sleep $(27.99 \pm 1.78 \mathrm{~Hz}$ vs $10.21 \pm 0.75 \mathrm{~Hz}$ for awake vs NREM sleep, respectively; Wilcoxon signed-rank test, $p<0.0001)$. Ripples occurred more frequently during NREM sleep than during awake epochs (7.84 \pm 0.59 ripples/min vs $22.96 \pm 1.28$ ripples/min, respectively; Wilcoxon signedrank test, $p<0.0001)$. We then repeated the analysis as described above for all ripples, but subdivided ripples according to the behavioral state. For each of 49 MD MUA cases, the averaged MI was calculated for awake and sleep ripples, and the significance of modulation was defined based on the averaged MI value. During awake state, a significant rippleassociated MD suppression was present in 43 of 49 MD MUA cases $(87.8 \%$ ), while fewer cases (35 of 49 , or $71.4 \%$, showed significant MD modulation during NREM sleep; $\chi_{(1)}^{2}=4.91, p=$ $0.027)$. Furthermore, the MD suppression during awake state was significantly stronger than during NREM sleep when only significantly modulated MD cases were considered $(-2.49 \pm 0.21$ vs $-1.60 \pm 0.11$ for MI of ripples during awake state vs NREM sleep, respectively; Wilcoxon signed-rank test, $p=0.00057$ ).

Bidirectional modulation of MD activity during sleep ripples We next examined how MD MUA varied across individual ripples. To this end, for each data set, the ripple times were bootstrapped and the b-MI was computed for 100 randomly selected ripples. Then, the same procedure was repeated 5000 times (for details, see Materials and Methods). This analysis confirmed that $\mathrm{MD}$ inhibition accompanied the majority of b-MIs (78.10 \pm $0.28 \%$ ); however, some b-MIs were associated with either enhanced or unchanged MD MUA $(0.61 \pm 0.02 \%$ and $21.29 \pm$ $0.30 \%$, respectively; Fig. $6 A$ ). Subdividing ripples according to the behavioral state (awake vs sleep) revealed that the b-MIs were mostly negative during awake state, while both positive and negative b-MIs were obtained during NREM sleep (Fig. 6B). Specifically, a significant MD suppression was present in $88.39 \pm 4.27 \%$ of b-MIs during awake state versus $59.79 \pm 4.34 \%$ of b-MIs during NREM sleep. The MD enhanced activity around ripples was observed in $0.35 \pm 0.2 \%$ and $1.11 \pm 0.7 \%$ of b-MIs during awake state and sleep, respectively. The difference in the distribution of 
A

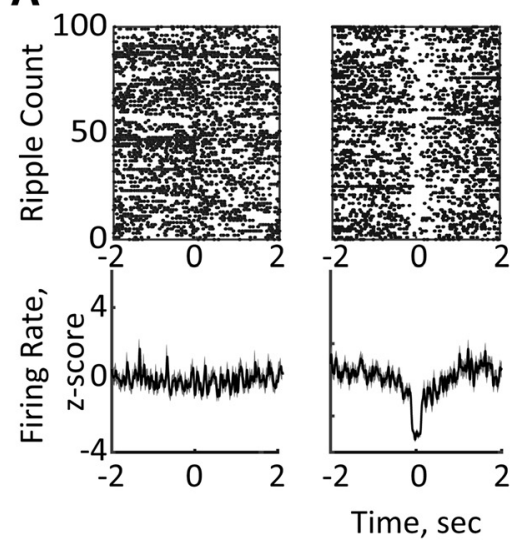

B

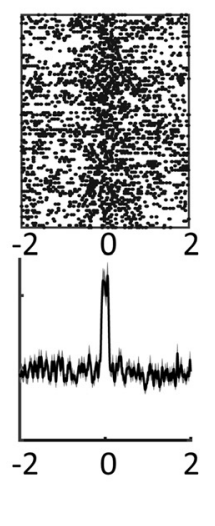

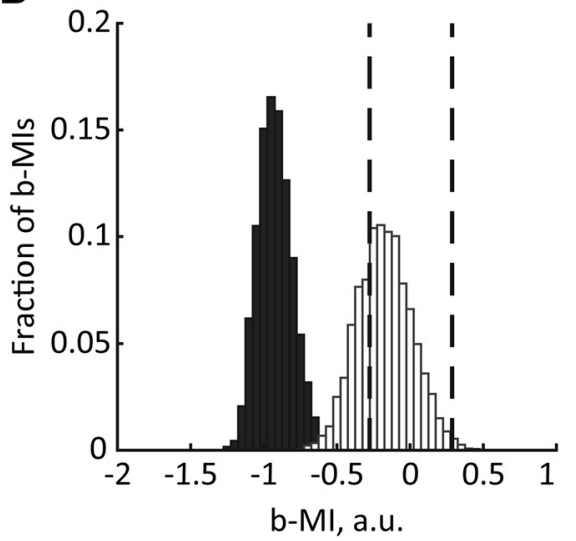

Figure 6. Ripple-associated MD modulation varies across behavioral states. $\boldsymbol{A}$, Bidirectional changes of MD MUA around ripples. Example raster plots (top) and corresponding averages of $z$-scored MD firing rates (bottom) are plotted around 100 randomly selected ripples. $\boldsymbol{B}$, Distribution of b-Mls generated by bootstrapping ripples during awake state (black) and NREM sleep (white). The vertical dashed lines mark the lower and upper limits of $95 \%$ confidence interval. Note that all b-Mls during awake state are negative, indicating consistent MD suppression, while both negative and positive b-Mls were obtained during NREM sleep.

b-MIs showing decreased, increased, or unmodulated MD MUA was statistically significant $\left(\chi_{(2)}^{2}=1064.99, p<0.0001\right)$. This result is consistent with a stronger $\mathrm{MD}$ suppression around awake ripples as revealed by MI.

\section{The MD activity is not suppressed around ripples co-occurring with sleep spindles}

Besides fluctuations of neural activity in the HPC and MD thalamus across awake and sleep states, a temporal coupling exists between the hippocampal ripples and sleep spindles, which is thought to enable the hippocampal-cortical information transfer underlying memory consolidation (Siapas and Wilson, 1998; Maingret et al., 2016; Latchoumane et al., 2017). It is also well established that sleep spindles emerge as a result of synchronized interplay within the thalamocortical circuit (Contreras and Steriade, 1997; Llinás and Steriade, 2006). Sleep spindles were detected during NREM sleep episodes as described previously (Novitskaya et al., 2016), and the times of spindle onsets and offsets were extracted. We first split ripples occurring during awake state $(34.3 \pm 2.1 \%)$ from the remaining ripples occurring during NREM sleep, and the latter were further subdivided into spindle-uncoupled $(51.8 \pm 0.7 \%)$ and spindle-coupled (13.9 \pm $0.6 \%$ ) ripples. Figure $7 A-C$ shows representative epochs depicting different patterns of ripple/spindle coupling. Ripples occurring during different behavioral/brain states differ by their intrinsic properties. We compared the intraripple frequency and the ripple amplitude among three patterns of event occurrence. The repeated-measures ANOVA revealed a significant effect of the condition for both the intraripple frequency $\left(F_{(2,181.11)}=\right.$ 4117.99, $p<0.0001)$ and the ripple amplitude $\left(F_{(2,164.57)}=\right.$ $1119.10, p<0.0001)$. The subsequent post hoc comparisons (Bonferroni corrected) showed that awake ripples had the highest intraripple frequency and the highest amplitude, while the spindlecoupled ripples had the lowest intraripple frequency and amplitude (Fig. 8). Table 1 summarizes the correlation strength between the ripple amplitude and MI for different subtypes of ripples.

We thus studied whether the ripple-associated MD activity depended on ongoing hippocampal-cortical population dynamics. Remarkably, two distinct patterns of MD MUA were observed during NREM sleep around ripples that were coupled or uncoupled with sleep spindles (Fig. $7 E, F$ ). Specifically, around spindle-uncoupled ripples, the MD was primarily suppressed
( $85.71 \%, n=42$ ), whereas no change ( 24 of 49 cases, $48.98 \%$ ) or a transient increase ( 20 of 49 cases, $40.82 \%$ ) of the firing rate was present around spindle-coupled ripples (Fig. $6 F$ ). The profile of MD MUA was almost identical around ripple-coupled spindles and around all detected sleep spindles (Fig. 9). Since during NREM sleep the MD suppression was predominant during spindle-uncoupled ripples as it was during awake ripples, we compared the magnitude of MD suppression between these two subsets of ripples. The MD suppression was significantly stronger around awake ripples (mean MI, $-2.46 \pm 0.20$ vs $-1.97 \pm 0.11$ for awake vs spindle-uncoupled sleep ripples, respectively; Wilcoxon signed-rank test, $p=0.02$ ).

\section{Discussion}

In the present study, we have characterized neural activity in the $\mathrm{MD}$, an associative thalamic nucleus, during epochs of memory replay as indicated by hippocampal ripples (Skelin et al., 2018). Overall, the MD spiking was transiently suppressed around both awake and sleep ripples. Notably, a decrease of MD firing rate systematically preceded the ripple onset. A stronger MD suppression corresponded to the ripples with higher amplitude and ones occurring sparsely (not in clusters). Strikingly, periripple MD modulation was fine-tuned to the thalamocortical activity dynamics such that the MD suppression was observed around ripples occurring outside sleep spindles. In contrast, during spindle-coupled ripples, no MD suppression was present, and in about half of cases, the MD firing was increased. We also characterized the MD activity around other hippocampal oscillatory patterns, namely, the high-gamma episodes and mixed gamma/ ripple oscillations. High-gamma oscillations are another prominent pattern of the hippocampal population activity that typically occurs during alert behaviors (Colgin and Moser, 2010), but also during low arousal states including NREM sleep (Csicsvari et al., 1999; Sullivan et al., 2011). We found that the MD suppression was much weaker or absent around high-gamma oscillations. Furthermore, although MD suppression was systematically present around gamma/ripple events, the modulation magnitude was lower than around pure ripples. The latter result is in agreement with existing evidence that although ripples and high-gamma oscillations share similar mechanisms, the network effects of gamma oscillations are more local (Sullivan et al., 2011). Overall, our present study provides new evidence for coordinated activity 
A
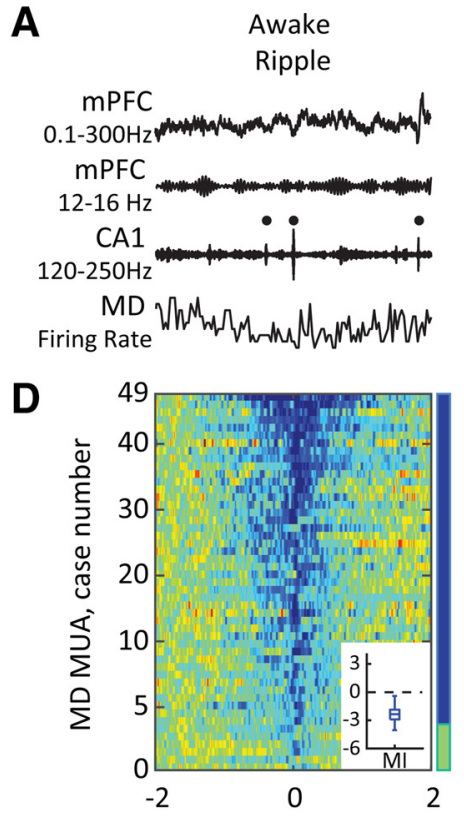

B

B Spindle-uncoupled Ripple

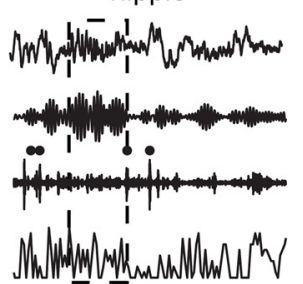

E

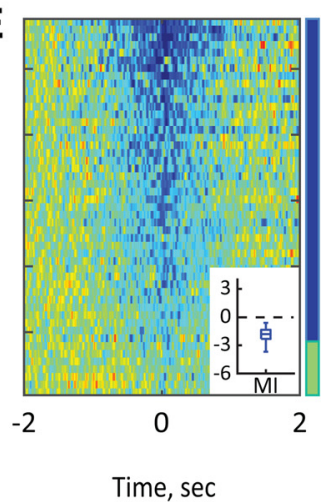

C

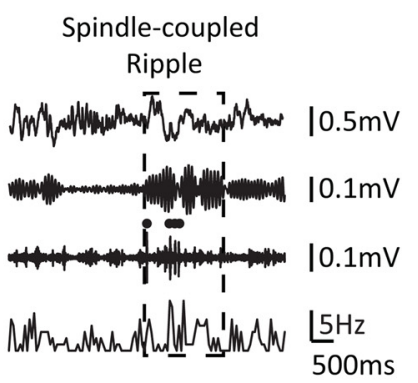

$\mathbf{F}$

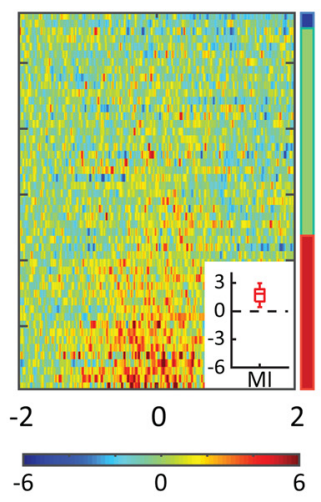

Figure 7. The MD activity is not suppressed around ripples co-occurring with sleep spindles. $A-C$, Top traces show the broad-band-filtered ( $0.1-300 \mathrm{~Hz})$ and bandpass-filtered (12-16 Hz) LFPs recorded from the $\mathrm{mPFC}$ and the bandpass-filtered ( $120-250 \mathrm{~Hz}$ ) LFPs recorded from the CA1 subfield of the dHPC during awake state (left) and NREM sleep (middle, right). Bottom traces show the corresponding MD firing rates. Black dots mark ripples, dashed-lined rectangles mark sleep spindles. $\boldsymbol{D}-\boldsymbol{F}$, Normalized firing rates of all MD MUA cases are plotted around ripple onset and sorted according to the MI value. The horizontal color bar shows z scores. Vertical color bars show the proportions of MD cases with significant decrease (blue), increase (red), or no change (green) in the firing rate. Insets, Box plots show the MIs distribution across MD MUA cases with significant firing rate modulation. Note that during NREM sleep, ripples may (right) or may not (middle) coincide with sleep spindles, and that periripple MD modulation depends on the ripple/spindle coupling.
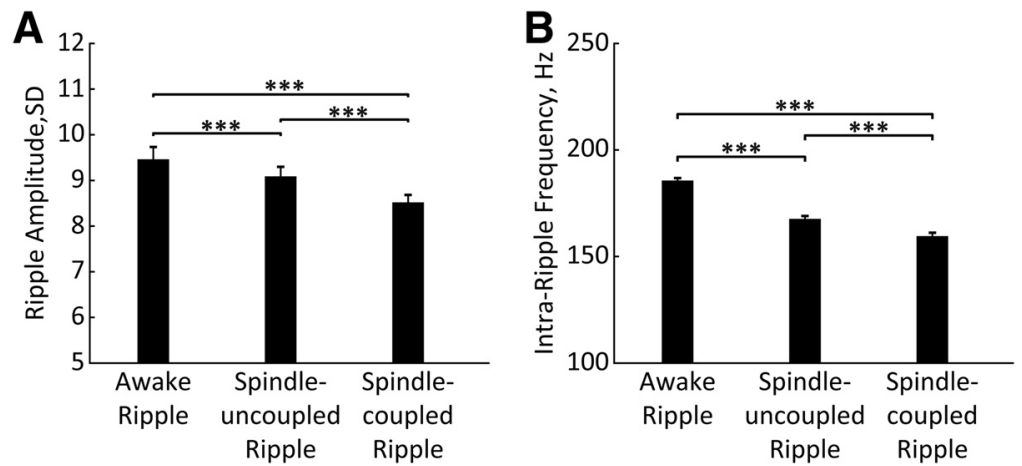

Figure 8. Intrinsic properties of different ripple types. $A, B$, Bars represent ripple amplitude $(\boldsymbol{A})$ and intraripple frequency $(\boldsymbol{B})$ for awake, spindle-uncoupled, and spindle-coupled ripples. ${ }^{* * *} p<0.001$ (Bonferroni corrected). Error bars indicate SE.

Table 1. MI of the MD MUA and MI correlation ( $r$ ) with different subsets of ripples

\begin{tabular}{|c|c|c|c|c|c|}
\hline & $\begin{array}{l}\text { First } \\
\text { quartile }\end{array}$ & $\begin{array}{l}\text { Second } \\
\text { quartile }\end{array}$ & $\begin{array}{l}\text { Third } \\
\text { quartile }\end{array}$ & $\begin{array}{l}\text { Fourth } \\
\text { quartile }\end{array}$ & $\begin{array}{l}\text { Correlation } \\
\text { coefficient }\end{array}$ \\
\hline MI, all ripples & $-1.11 \pm 0.10$ & $-1.57 \pm 0.11$ & $-1.80 \pm 0.17$ & $-1.81 \pm 0.15$ & $r=-0.28^{* * *}$ \\
\hline MI, awake ripples & $-0.94 \pm 0.10$ & $-1.26 \pm 0.13$ & $-1.35 \pm 0.11$ & $-1.30 \pm 0.11$ & $r=-0.23^{* * *}$ \\
\hline $\begin{array}{l}\text { MI, spindle- } \\
\text { uncoupled ripples }\end{array}$ & $-0.93 \pm 0.09$ & $-1.30 \pm 0.13$ & $-1.20 \pm 0.11$ & $-1.20 \pm 0.06$ & $r=-0.12^{*}$ \\
\hline $\begin{array}{l}\text { MI, spindle- } \\
\text { coupled ripples }\end{array}$ & $0.58 \pm 0.10$ & $0.46 \pm 0.10$ & $0.41 \pm 0.09$ & $0.45 \pm 0.05$ & $r=-0.06$ \\
\hline
\end{tabular}

between the associative thalamus and the dHPC during off-line states and raises further questions about causality of these interactions and the functional significance of the ripple-associated modulation of MD neural activity.
Brain-wide activity pattern associated with hippocampal ripples

By now, it is well established that many cortical regions show coordinated firing with hippocampal population bursts (Sirota et al., 2003; Ji and Wilson, 2007; Peyrache et al., 2009; Wierzynski et al., 2009; Wang and Ikemoto, 2016; Rothschild et al., 2017; Wilber et al., 2017). Inhibition of mPFC neurons was documented around awake ripples (Jadhav et al., 2016). Bidirectional modulation of prefrontal neurons during memory replay is well suited for selectivity of information transfer and triggering synaptic plasticity within memory trace-specific cell assemblies (Peyrache et al., 2011; Jadhav et al., 2016). Ripple-associated neuron firing was also shown in the ventral striatum (Lansink et al., 2008). Both excitatory and inhibitory responses during ripples were observed in the VTA (Gomperts et al., 2015) and BLA neurons (Girardeau et al., 2017). Our previous study combining electrophysiological recordings in the HPC with fMRI-based whole-brain activity mapping provided the first evidence for inverse relationships between thalamic and hippocampal activity during ripples (Logothetis et al., 2012). The ripple-associated spiking suppression was subsequently demonstrated in the lateral geniculate nucleus in nonhuman primates (Logothetis, 2015). Similar findings have been reported previously in rats for the midline thalamus (Lara-Vásquez et al., 2016) and the median raphe nucleus (Wang et al., 2015). Together, these observations reflect a widespread activation/inhibition pattern of brain activity associated with presumed memory replay as indicated by hip- 
pocampal ripples. At present, the specificity of such distributed brain activity and its relevance to memory consolidation remains poorly understood.

\section{Bidirectional modulation of ripple- associated thalamic activity}

The main finding of the present study is differential engagement of the MD during spindle-coupled versus spindle-uncoupled ripples. The elevated firing rate of MD neurons during spindle-coupled ripples is not surprising. It is well established that sleep spindles emerge due to coordinated interactions within the thalamocortical circuit (Contreras et al., 1996; Steriade, 2006). It has also been demonstrated that ripples tend to occur during periods of enhanced cortical excitability, or up states (Sirota et al., 2003; Battaglia et al., 2004; Mölle et al., 2006), and coincide with sleep spindles (Siapas and Wilson, 1998). In our recordings, about $20 \%$ of ripples were temporally coupled with sleep spindles. Expectedly, the MD activity was enhanced during spindles, regardless of whether spindles coincided with ripples or not. A reduced inhibitory input from the limbic-projecting thalamic reticular nucleus (TRN) during sleep spindles (Halassa et al., 2014) is likely the key component regulating this spindleassociated MD activation. On the other hand, one-half of sleep ripples and all awake ripples were not temporally coupled with spindles, and those spindle-uncoupled ripples ( $~ 80 \%$ of all ripples) were consistently associated with pronounced MD suppression. Thus, we observed a synergistic activity within the thalamic-hippocampal-cortical network during time windows of ripple/spindle coupling, yet a memory-specific relevance of the MD coactivation is currently unknown.

Spindle-ripple coupling has been suggested to be critical for memory consolidation (Diekelmann and Born, 2010). Indeed, an experimentally induced increase of spindle-ripple coupling during postlearning sleep improved memory (Maingret et al., 2016; Latchoumane et al., 2017), while its disturbance impaired memory (Novitskaya et al., 2016). Hippocampal ripples tend to precede sleep spindles (Siapas and Wilson, 1998; Wierzynski et al., 2009; Peyrache et al., 2011); therefore, memory trace reactivation during ripples may trigger synaptic modifications in the cortex during subsequent spindles (Johnson et al., 2010; Timofeev and Chauvette, 2017). It was hypothesized that the hippocampal output during replay may selectively recruit thalamocortical cells into thalamocortical rhythmic interactions reflected as spindles (Peyrache et al., 2011). A strong engagement of cortical inhibition during spindles may permit activation of cortical cell assemblies representing specific memory traces and may therefore reinforce synaptic plasticity within a selective network (Peyrache et al., 2011; Jadhav et al., 2016). The MD coactivation during ripplespindle coupling may thus facilitate long-range network interactions (Nakajima and Halassa, 2017).

The thalamic suppression during spindle-uncoupled ripples may be favorable for memory processing, as it appears to reduce the thalamocortical relay of sensory information. Indeed, $\mathrm{mPFC}$ neurons appear more responsive to hippocampal input during spindle-uncoupled ripples, that is, during periods when the recurrent thalamocortical circuit is not engaged (Peyrache et al., 2011). In this context, a source of MD inhibition presents an interesting question. The ripple-associated MD modulation does not appear to simply reflect global fluctuations of thalamocorti- cal activity that is largely controlled by the TRN (Steriade et al., 1993). Extrathalamic inhibitory control of the thalamic activity is thought to be more selective and therefore more effective for state-dependent gating of thalamocortical information transfer (Bokor et al., 2005; Halassa and Acsády, 2016). For example, cholinergic inputs from the midbrain parabrachial region cause hyperpolarization in the thalamocortical neurons in auditory thalamus (Mooney et al., 2004). Another candidate is the GABAergic input from the zona incerta that selectively targets higher-order thalamic relays (Bokor et al., 2005). The precise temporal dynamics of these interactions would shed light on the neurophysiological mechanisms controlling coordinated activation/deactivation of competing large-scale networks.

The strength of long-range interactions may depend on the behavioral state and cognitive context. For example, a previous study reported differential modulation of activity in the anterior cingulate cortex (ACC) around awake and sleep ripples (Wang and Ikemoto, 2016). Wang and Ikemoto (2016) proposed that the absence of ACC activation during awake ripples may protect freshly acquired information from alteration and, in turn, facilitate intrahippocampal information processing. Furthermore, a type of MD interaction with the hippocampal-cortical network may gate the direction of information transfer. It has been shown that during time windows of spindle-ripple coupling (and MD coactivation), the cortex is less responsive to hippocampal input (Peyrache et al., 2011); therefore, cortical signaling to the HPC may prevail. In turn, during spindle-uncoupled ripples (and MD inhibition), input from the HPC to the cortex is prevalent. Evidence accumulates that awake ripples may additionally support cognitive functions, such as behavioral planning and decision making (Diba and Buzsáki, 2007; Yu and Frank, 2015). Therefore, the MD suppression around awake ripples may also favor memory retrieval from cortex or other nonmnemonic functions requiring corticalhippocampal interactions. Thus, the functional state of the thalamic-hippocampal-cortical network as well as the content of neural replay and directionality of information transfer may differ during awake, spindle-coupled, and spindle-uncoupled ripples, and these intriguing possibilities shall be further explored.

\section{Possible role of the MD for declarative memory}

The anatomical connectivity of the MD supports its involvement in processing and integration of diverse information and mediating it to the mPFC (Mitchell, 2015; Wolff et al., 2015). Consequently, the MD may present a key element within the executive control circuit (Van Der Werf et al., 2003; Ketz et al., 2015), which in the case of declarative memory includes the HPC. Through its connectivity with the hippocampal input and output 
structures (Groenewegen, 1988; Burwell, 2000), the MD can mediate the bidirectional information flow between the HPC and mPFC (Floresco and Grace, 2003; Peyrache et al., 2011). Consistent with the thalamic control of sensory selection (Saalmann and Kastner, 2011), the MD neuronal populations may coordinate selective engagement of functionally diverse neural circuits in particular oscillatory dynamics (Ketz et al., 2015). Indeed, the MD-PFC synchronization within the $\beta$ (13-30 Hz) frequency range accompanied performance of a spatial memory task (Parnaudeau et al., 2013). Moreover, the MD inactivation reduced MD-PFC $\beta$ synchronization and also impaired the task performance (Parnaudeau et al., 2013). Furthermore, sustained activity within the MD-PFC network has been implicated in maintaining information in working memory (Bolkan et al., 2017; Schmitt et al., 2017). Thus, the MD may enhance functional connectivity within a specific brain network (Nakajima and Halassa, 2017).

MD involvement in memory consolidation has been suggested (Squire, 1986; Lee et al., 2011; Tu et al., 2014; LaraVásquez et al., 2016), yet this hypothesis requires further experimental validation. The fact that a decrease in MD firing rate systematically preceded the ripple onset suggests that a decreased spiking in the MD may be related to reorganization of the network activity and a shift to a brain state permitting ripple generation, which in turn promotes the hippocampal-cortical communication mediating declarative memory consolidation. Apart from the MD, the midline thalamic nuclei have also been considered to play a role for memory processes (Pereira de Vasconcelos and Cassel, 2015). For example, based on anatomical connectivity, the nucleus reuniens could mediate the prefrontalhippocampal interactions (Varela et al., 2014). A previous study by Lara-Vásquez et al. (2016) correlated the activity of midline thalamic neurons with different oscillations in the HPC and observed a functional heterogeneity among different cell types. Specifically, calcium-binding protein calretinin (CR)-negative neurons were modulated by theta oscillations, while CR-positive neurons were inhibited during hippocampal ripples. In our study, we sampled a relatively small number of MD single units and did not observe differential modulation. However, heterogeneity of MD responses may be revealed in the future by examining a larger population of MD single units.

The thalamoprefrontal and hippocampal-prefrontal pathways appear to participate in different types of information processing (Ketz et al., 2015; Eichenbaum, 2017), and, therefore, coactivation of these functionally distinct networks may lead to interference. If, indeed, the MD belongs to a competing (e.g., sensory-mediating) network, its activation during ripples would likely interfere with the stabilization of recent memory traces. Thus, a decrease in MD activity may reflect inactivation of a competing network, which would in turn facilitate the hippocampal-cortical communication mediating declarative memory consolidation. Consistent with this view, we showed previously (Novitskaya et al., 2016) that thalamic activation produced by ripple-triggered Locus Coeruleus stimulation impaired consolidation of spatial memory in rats. Our present findings provide evidence that a transient suppression of MD neural activity that precedes hippocampal ripples may possibly play an active role in coordinating activity within large-scale networks involved in different aspects of information processing taking place off-line.

Thus, the activity pattern within a given brain region may reflect its engagement or disengagement within a functionally distinct and distributed network enabling specific types of information processing. Bidirectional modulation of MD activity around ripples was linked to different dynamic states of the thala- mic-hippocampal-cortical network differing by topography, the directionality of information transfer, and the brain functions they support. Possibly, spindle-uncoupled ripples combined with thalamic activity suppression presents a brain state that is favorable for memory replay and information transfer from the HPC to the cortex, while the thalamic input at times of hippocampal-cortical communication, indicated by spindle/ripple coupling, may contribute to the neural assembly selection for promoting synaptic plasticity in the cortex. Our present findings open questions about the neurophysiological mechanisms regulating these fine-tuned, thalamic-hippocampal-cortical interactions and their functional significance.

\section{References}

Battaglia FP, Sutherland GR, McNaughton BL (2004) Hippocampal sharp wave bursts coincide with neocortical "up-state" transitions. Learn Memory 11:697-704. CrossRef

Bokor H, Frère SG, Eyre MD, Slézia A, Ulbert I, Lüthi A, Acsády L (2005) Selective GABAergic control of higher-order thalamic relays. Neuron 45: 929-940. CrossRef Medline

Bolkan SS, Stujenske JM, Parnaudeau S, Spellman TJ, Rauffenbart C, Abbas AI, Harris AZ, Gordon JA, Kellendonk C (2017) Thalamic projections sustain prefrontal activity during working memory maintenance. Nat Neurosci 20:987-996. CrossRef Medline

Burwell RD (2000) The parahippocampal region: corticocortical connectivity. Ann N Y Acad Sci 911:25-42. Medline

Buzsáki G (1989) Two-stage model of memory trace formation: a role for "noisy" brain states. Neuroscience 31:551-570. CrossRef Medline

Buzsáki G (2004) Large-scale recording of neuronal ensembles. Nat Neurosci 7:446-451. CrossRef Medline

Chrobak JJ, Buzsáki G (1996) High-frequency oscillations in the output networks of the hippocampal-entorhinal axis of the freely behaving rat. J Neurosci 16:3056-3066. CrossRef Medline

Colgin LL, Moser EI (2010) Gamma oscillations in the hippocampus. Physiology 25:319-329. CrossRef Medline

Contreras D, Steriade M (1997) Synchronization of low-frequency rhythms in corticothalamic networks. Neuroscience 76:11-24. Medline

Contreras D, Destexhe A, Sejnowski TJ, Steriade M (1996) Control of spatiotemporal coherence of a thalamic oscillation by corticothalamic feedback. Science 274:771-774. CrossRef Medline

Csicsvari J, Hirase H, Czurkó A, Mamiya A, Buzsáki G (1999) Fast network oscillations in the hippocampal CA1 region of the behaving rat. J Neurosci 19:RC20. CrossRef Medline

Diba K, Buzsáki G (2007) Forward and reverse hippocampal place-cell sequences during ripples. Nat Neurosci 10:1241-1242. CrossRef Medline

Diekelmann S, Born J (2010) The memory function of sleep. Nat Rev 11: 114-126. CrossRef

Eichenbaum H (2017) Prefrontal-hippocampal interactions in episodic memory. Nat Revi 18:547-558. CrossRef

Floresco SB, Grace AA (2003) Gating of hippocampal-evoked activity in prefrontal cortical neurons by inputs from the mediodorsal thalamus and ventral tegmental area. J Neurosci 23:3930-3943. CrossRef Medline

Girardeau G, Inema I, Buzsáki G (2017) Reactivations of emotional memory in the hippocampus-amygdala system during sleep. Nat Neurosci 20:1634-1642. CrossRef Medline

Gomperts SN, Kloosterman F, Wilson MA (2015) VTA neurons coordinate with the hippocampal reactivation of spatial experience. Elife 4:e05360. CrossRef Medline

Groenewegen HJ (1988) Organization of the afferent connections of the mediodorsal thalamic nucleus in the rat, related to the mediodorsalprefrontal topography. Neuroscience 24:379-431. CrossRef Medline

Halassa MM, Acsády L (2016) Thalamic inhibition: diverse sources, Diverse Scales. Trends Neurosci 39:680-693. CrossRef Medline

Halassa MM, Chen Z, Wimmer RD, Brunetti PM, Zhao S, Zikopoulos B, Wang F, Brown EN, Wilson MA (2014) State-dependent architecture of thalamic reticular subnetworks. Cell 158:808-821. CrossRef Medline

Jadhav SP, Rothschild G, Roumis DK, Frank LM (2016) Coordinated excitation and inhibition of prefrontal ensembles during awake hippocampal sharp-wave ripple events. Neuron 90:113-127. CrossRef Medline

Jay TM, Thierry AM, Wiklund L, Glowinski J (1992) Excitatory amino acid pathway from the hippocampus to the prefrontal cortex. contribution of 
AMPA receptors in hippocampo-prefrontal cortex transmission. Eur J Neurosci 4:1285-1295. CrossRef Medline

Ji D, Wilson MA (2007) Coordinated memory replay in the visual cortex and hippocampus during sleep. Nat Neurosci 10:100-107. CrossRef Medline

Johnson LA, Euston DR, Tatsuno M, McNaughton BL (2010) Stored-trace reactivation in rat prefrontal cortex is correlated with down-to-up state fluctuation density. J Neurosci 30:2650-2661. CrossRef Medline

Kapoor V, Krampe E, Klug A, Logothetis NK, Panagiotaropoulos TI (2013) Development of tube tetrodes and a multi-tetrode drive for deep structure electrophysiological recordings in the macaque brain. J Neurosci Methods 216:43-48. CrossRef Medline

Ketz NA, Jensen O, O'Reilly RC (2015) Thalamic pathways underlying prefrontal cortex-medial temporal lobe oscillatory interactions. Trends Neurosci 38:3-12. CrossRef Medline

Lansink CS, Goltstein PM, Lankelma JV, Joosten RN, McNaughton BL, Pennartz CM (2008) Preferential reactivation of motivationally relevant information in the ventral striatum. J Neurosci 28:6372-6382. CrossRef Medline

Lara-Vásquez A, Espinosa N, Durán E, Stockle M, Fuentealba P (2016) Midline thalamic neurons are differentially engaged during hippocampus network oscillations. Sci Rep 6:29807. CrossRef Medline

Latchoumane CV, Ngo HV, Born J, Shin HS (2017) Thalamic spindles promote memory formation during sleep through triple phase-locking of cortical, thalamic, and hippocampal rhythms. Neuron 95:424-435.e6. CrossRef Medline

Lee S, Ahmed T, Lee S, Kim H, Choi S, Kim DS, Kim SJ, Cho J, Shin HS (2011) Bidirectional modulation of fear extinction by mediodorsal thalamic firing in mice. Nat Neurosci 15:308-314. Medline

Llinás RR, Steriade M (2006) Bursting of thalamic neurons and states of vigilance. J Neurophysiol 95:3297-3308. CrossRef Medline

Logothetis NK (2015) Neural-event-triggered fMRI of large-scale neural networks. Curr Opin Neurobiol 31:214-222. CrossRef Medline

Logothetis NK, Eschenko O, Murayama Y, Augath M, Steudel T, Evrard HC, Besserve M, Oeltermann A (2012) Hippocampal-cortical interaction during periods of subcortical silence. Nature 491:547-553. CrossRef Medline

Maingret N, Girardeau G, Todorova R, Goutierre M, Zugaro M (2016) Hippocampo-cortical coupling mediates memory consolidation during sleep. Nat Neurosci 19:959-964. CrossRef Medline

Markowitsch HJ (1982) Thalamic mediodorsal nucleus and memory: a critical evaluation of studies in animals and man. Neurosci Biobehav Rev 6:351-380. CrossRef Medline

Mitchell AS (2015) The mediodorsal thalamus as a higher order thalamic relay nucleus important for learning and decision-making. Neurosci Biobehav Rev 54:76-88. CrossRef Medline

Mölle M, Yeshenko O, Marshall L, Sara SJ, Born J (2006) Hippocampal sharp wave-ripples linked to slow oscillations in rat slow-wave sleep. J Neurophysiol 96:62-70. CrossRef Medline

Mooney DM, Zhang L, Basile C, Senatorov VV, Ngsee J, Omar A, Hu B (2004) Distinct forms of cholinergic modulation in parallel thalamic sensory pathways. Proc Natl Acad Sci U S A 101:320-324. CrossRef Medline

Nakajima M, Halassa MM (2017) Thalamic control of functional cortical connectivity. Curr Opin Neurobiol 44:127-131. CrossRef Medline

Novitskaya Y, Sara SJ, Logothetis NK, Eschenko O (2016) Ripple-triggered stimulation of the locus coeruleus during post-learning sleep disrupts ripple/spindle coupling and impairs memory consolidation. Learn Memory 23:238-248. CrossRef

Parnaudeau S, O'Neill PK, Bolkan SS, Ward RD, Abbas AI, Roth BL, Balsam PD, Gordon JA, Kellendonk C (2013) Inhibition of mediodorsal thalamus disrupts thalamofrontal connectivity and cognition. Neuron 77: 1151-1162. CrossRef Medline

Pereira de Vasconcelos A, Cassel J-C (2015) The nonspecific thalamus: A place in a wedding bed for making memories last? Neuroscience \& Biobehavioral Reviews 54:175-196. CrossRef Medline

Peyrache A, Khamassi M, Benchenane K, Wiener SI, Battaglia FP (2009) Replay of rule-learning related neural patterns in the prefrontal cortex during sleep. Nat Neurosci 12:919-926. CrossRef Medline

Peyrache A, Battaglia FP, Destexhe A (2011) Inhibition recruitment in prefrontal cortex during sleep spindles and gating of hippocampal inputs. Proc Natl Acad Sci U S A 108:17207-17212. CrossRef Medline

Ramirez-Villegas JF, Logothetis NK, Besserve M (2015) Diversity of sharp- wave-ripple LFP signatures reveals differentiated brain-wide dynamical events. Proc Natl Acad Sci U S A 112:E6379-E6387. CrossRef Medline

Rothschild G, Eban E, Frank LM (2017) A cortical-hippocampal-cortical loop of information processing during memory consolidation. Nat Neurosci 20:251-259. Medline

Saalmann YB, Kastner S (2011) Cognitive and perceptual functions of the visual thalamus. Neuron 71:209-223. CrossRef Medline

Schmitt LI, Wimmer RD, Nakajima M, Happ M, Mofakham S, Halassa MM (2017) Thalamic amplification of cortical connectivity sustains attentional control. Nature 545:219-223. CrossRef Medline

Selinger JV, Kulagina NV, O’Shaughnessy TJ, Ma W, Pancrazio JJ (2007) Methods for characterizing interspike intervals and identifying bursts in neuronal activity. J Neurosci Methods 162:64-71. CrossRef Medline

Siapas AG, Wilson MA (1998) Coordinated interactions between hippocampal ripples and cortical spindles during slow-wave sleep. Neuron 21:1123-1128. CrossRef Medline

Sirota A, Csicsvari J, Buhl D, Buzsáki G (2003) Communication between neocortex and hippocampus during sleep in rodents. Proc Natl Acad Sci U S A 100:2065-2069. CrossRef Medline

Skelin I, Kilianski S, McNaughton BL (2018) Hippocampal coupling with cortical and subcortical structures in the context of memory consolidation. Neurobiol Learn Memory. Advance online publication. Retrieved April 13, 2018. doi: 10.1016/j.nlm.2018.04.004.

Squire LR (1986) Mechanisms of memory. Science 232:1612-1619. CrossRef Medline

Steriade M (2006) Grouping of brain rhythms in corticothalamic systems. Neuroscience 137:1087-1106. CrossRef Medline

Steriade M, McCormick DA, Sejnowski TJ (1993) Thalamocortical oscillations in the sleeping and aroused brain. Science 262:679-685. CrossRef Medline

Sullivan D, Csicsvari J, Mizuseki K, Montgomery S, Diba K, Buzsáki G (2011) Relationships between hippocampal sharp waves, ripples, and fast gamma oscillation: influence of dentate and entorhinal cortical activity. J Neurosci 31:8605-8616. CrossRef Medline

Timofeev I, Chauvette S (2017) Sleep slow oscillation and plasticity. Curr Opin Neurobiol 44:116-126. CrossRef Medline

Tu S, Miller L, Piguet O, Hornberger M (2014) Accelerated forgetting of contextual details due to focal medio-dorsal thalamic lesion. Front Behav Neurosci 8:320. Medline

Van Der Werf YD, Jolles J, Witter MP, Uylings HBM (2003) Contributions of thalamic nuclei to declarative memory functioning. Cortex 39:10471062. CrossRef Medline

Varela C, Kumar S, Yang JY, Wilson MA (2014) Anatomical substrates for direct interactions between hippocampus, medial prefrontal cortex, and the thalamic nucleus reuniens. Brain Struct Funct 219:911-929. CrossRef Medline

Wang DV, Ikemoto S (2016) Coordinated interaction between hippocampal sharp-wave ripples and anterior cingulate unit activity. J Neurosci 36:10663-10672. CrossRef Medline

Wang DV, Yau HJ, Broker CJ, Tsou JH, Bonci A, Ikemoto S (2015) Mesopontine median raphe regulates hippocampal ripple oscillation and memory consolidation. Nat Neurosci 18:728-735. Medline

Wang SH, Morris RG (2010) Hippocampal-neocortical interactions in memory formation, consolidation, and reconsolidation. Annual review of psychology 61:49-79, C1-C4. CrossRef Medline

Wierzynski CM, Lubenov EV, Gu M, Siapas AG (2009) State-dependent spike-timing relationships between hippocampal and prefrontal circuits during sleep. Neuron 61:587-596. CrossRef Medline

Wilber AA, Skelin I, Wu W, McNaughton BL (2017) Laminar organization of encoding and memory reactivation in the parietal cortex. Neuron 95: 1406-1419.e5. CrossRef Medline

Wolff M, Alcaraz F, Marchand AR, Coutureau E (2015) Functional heterogeneity of the limbic thalamus: from hippocampal to cortical functions. Neurosci Biobehav Rev 54:120-130. CrossRef Medline

Ylinen A, Bragin A, Nádasdy Z, Jandó G, Szabó I, Sik A, Buzsáki G (1995) Sharp wave-associated high-frequency oscillation $(200 \mathrm{~Hz})$ in the intact hippocampus: network and intracellular mechanisms. J Neurosci 15:30 46. CrossRef Medline

Yu JY, Frank LM (2015) Hippocampal-cortical interaction in decision making. Neurobiol Learn Memory 117:34-41. CrossRef 